\title{
Investigation on dimensional accuracy and mechanical properties of cylindrical parts by flow forming
}

\author{
Gangfeng Xiao ${ }^{1}$, Qinxiang Xia ${ }^{1}$, Xiuquan Cheng ${ }^{2}$, and Hui Long ${ }^{3}$ \\ ${ }^{1}$ School of Mechanical and Automotive Engineering, South China University of Technology, \\ Guangzhou 510641, China \\ ${ }^{2}$ School of Aircraft Maintenance Engineering, Guangzhou Civil Aviation College, Guangzhou \\ 510403, Guangdong, China \\ ${ }^{3}$ Department of Mechanical Engineering, the University of Sheffield, Sheffield S1 3JD, UK
}

\begin{abstract}
The high dimensional accuracy and excellent mechanical properties have become two most important requirements for structural components. In this paper, experiments using two spinning methods, stagger spinning and counter-roller spinning, were carried out under different thinning ratio of wall thickness of spun parts. The influence of spinning methods and total thinning ratio of wall thickness on the dimensional accuracy and mechanical properties of the!spun parts were studied. It shows that the wall thickness deviation and ovality of the spun parts are closely related to the spinning method and the total thinning ratio of wall thickness. The hardness of the spun parts increases with the increasing of the total thinning ratio, and the hardness along the thickness direction of the spun parts manufactured by counter-roller spinning is more homogeneous than that of the stagger spinning. The strength and the elongation of the spun parts are mainly influenced by the total thinning ratio, with little relevance to the spinning method.
\end{abstract}

\section{Introduction}

Cylindrical parts are one of the important structural components which are widely used in various industrial sectors. In some industrial applications, the structural components have to operate in harsh environment with heavy-load, high temperature and pressure; thus the high dimensional accuracy and excellent mechanical properties have become two most important requirements for structural components [1].

Metal spinning processes belong to near net-shape forming technology. According to the deformation characteristics of the blank material, spinning can be divided into conventional spinning and power spinning [2]. Flow forming, which belongs to power spinning, is one of the most effective methods to manufacture cylindrical parts. It mainly includes the stagger spinning and counter-roller spinning [3]. During stagger spinning, the thinning ratio of thickness in one pass is divided among three rollers, the productivity is improved considerably, thus the processing method has been widely used in

This is an Open Access article distributed under the terms of the Creative Commons Attribution License 4.0, which permits unrestricted use, distribution, and reproduction in any medium, provided the original work is properly cited. 


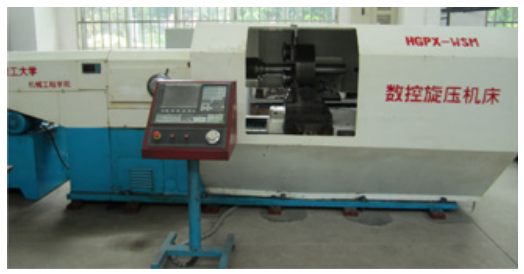

Figure 1. HGPX-WSM Type CNC spinning machine.

aviation, aerospace and automotive industries [4]. During counter-roller spinning, however, the mandrel is replaced by the inner rollers and the spinning force is reduced to $50 \%$ of that in stagger spinning, it is particularly suitable for manufacturing large dimensional cylindrical parts and has attracted significant research interests in recent investigations [3].

Grain refinement is the most important way to improve the overall mechanical properties of the structural components, especially when the grains are refined to the nanoscale [5]. Xia et al. have manufactured cylindrical parts with nano/ultrafine grain structure by stagger spinning and counter-roller spinning respectively, and the mean grain size has been refined from $50 \mu \mathrm{m}$ to $600 \mathrm{~nm}$ successfully $[4,6]$. They also reported that the value of LSP (layer spacing of pearlite) had been decreased with the increasing of the thinning ratio of the wall thickness during power spinning, and the tensile strength of spun tubes had been founded to be proportional to the reciprocal of LSP and the elongation was inversely proportional to the reciprocal of LSP [4]. Xiao et al. reported that the distribution of equivalent strains and the percentage of grain refinement of the spun parts obtained by counter-roller spinning were more homogeneous than that of the stagger spinning [3]. The distribution of the equivalent strains of the spun parts obtained by stagger spinning and counter-roller spinning was different, which affected the dimensional accuracy and mechanical properties of the spun parts $[3,4]$. To obtain the cylindrical parts with required mechanical properties and microstructure, the effect of different spinning methods and the total thinning ratio of wall thickness on the dimensional accuracy and mechanical properties of the spun parts are studied.

\section{Experimental investigation}

The cylindrical blanks used for the experiment are the annealed seamless ASTM 1020 steel tubes. The mechanical properties of the ASTM 1020 steel are the follows: yield strength $\sigma_{s}=325 \mathrm{MPa}$, tensile strength $\sigma_{\mathrm{s}}=535 \mathrm{MPa}$ and elongation $\delta=32 \sim 35 \%$, which are obtained by the uniaxial tension test at the room temperature according to the GB/T 228.1-2010 [7].

The experiment is carried out on the HGPX-WSM CNC spinning machine (Fig. 1). Figure 2 shows the principle of stagger spinning and counter-roller spinning. Figure 3 shows the devices used in the stagger spinning and the counter-roller spinning, respectively [3]. For flow forming, the main processing parameters influencing the dimensional accuracy and mechanical properties are the feed rate of roller $f$, per pass thinning ratio of wall thickness $\psi_{i}\left(\psi_{i}=\left(t_{i-1}-t_{i}\right) / t_{i-1}\right.$, where $t_{i-1}$ is the wall thickness of the cylindrical parts after $i-1$ passes spinning, $t_{i}$ is the wall thickness of the cylindrical parts after $i$ passes spinning), and axial offset $a$ and radial offset $b$ between rollers (Fig. 2a) [4]. According to Wang and Liu, for low-carbon steel, the feed rate $f$ should be in the range of $0.2-1.0 \mathrm{~mm} / \mathrm{r}$ [8]; considering both process efficiency and dimensional accuracy, the feed rate $f$ is selected as $0.6 \mathrm{~mm} / \mathrm{r}$ [9]. The optimum thinning ratio per pass $\psi_{i}$ is $30-45 \%$, as reported by Xia et al. [3]. To keep the radial force induced by each roller being equilibrated, the value of the axial offset $a$ and radial offset $b$ during stagger spinning can be selected as listed in Table 1, where the calculated values of the axial offset $a_{12}$ and $a_{23}$ are very close. The value of axial offset should be rounded to be easily implemented in the experiment, so the 


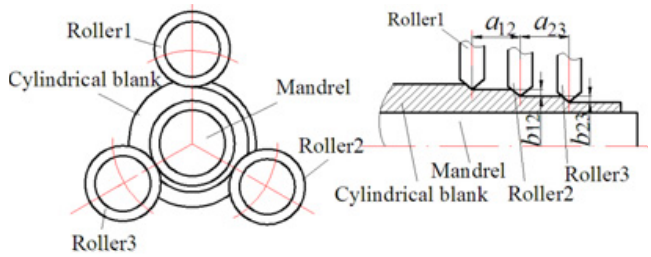

(a) Stagger spinning

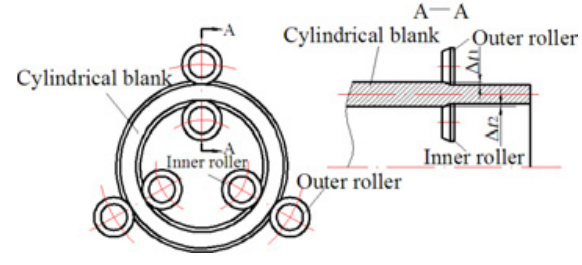

(b) Counte-roller spinning

Figure 2. Spinning methods [3].

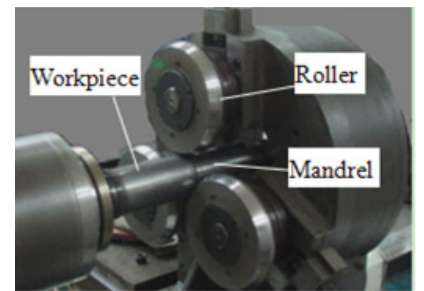

(a) Stagger spinning

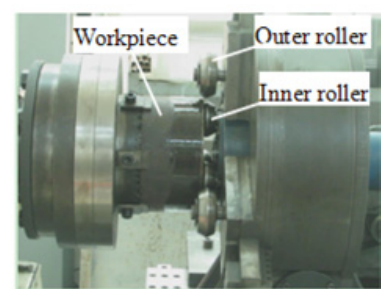

(b) Counte-roller spinning

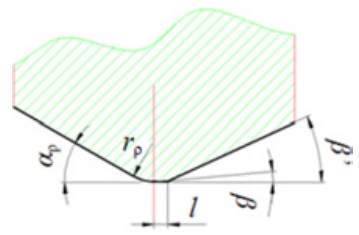

Figure 3. Spinning devices [3].

Figure 4. Schematic of roller structure [3].

values of the axial offset $a_{12}$ and $a_{23}$ can be selected as the same [4]. The rotational speed of mandrel $n$ has not shown obvious influence on the dimensional accuracy, as reported by Xia et al. [10], thus the rotational speed $n$ is selected as $108 \mathrm{r} / \mathrm{min}$ based on the capacity of the spinning machine [4]. The reduction $\Delta \mathrm{t}$ of the inner and outer rollers (Fig. 2b) during counter-roller spinning should be the same to obtain the homogeneous distribution of strains of the spun parts along the thickness direction [11]. Therefore, the main process parameters used in the experiments of stagger spinning and counter-roller spinning are selected as listed in Table 1 and Table 2, respectively.

In flow forming, the double-tapered roller (Fig. 4) is usually used for forming the cylindrical blank with the medium thickness [12]. The key geometric dimensions of rollers are as follows: forming angle $\alpha_{\rho}=25^{\circ}$, sleeking angle $\beta=3^{\circ}$, receding angle $\beta^{\prime}=30^{\circ}$, roundness radius $r_{\rho}=6 \mathrm{~mm}$, and polishing belt length $l=1.3 \mathrm{~mm}$ [3], as shown in Fig. 4. The diameter of rollers used in the stagger spinning is $D=180 \mathrm{~mm}$, while the diameter of rollers used in the counter-roller spinning is different from that of the stagger spinning. This is because that the three inner rollers must be installed inside the blank during the counter-roller spinning which are determined by the diameter of the cylindrical blank. Moreover, considering the consistence of contact areas between the inner and outer rollers and the blank, the diameters of inner and outer rollers used for the counter-roller spinning are selected as $40 \mathrm{~mm}$ and $60 \mathrm{~mm}$, respectively [13].

\section{Results and discussion}

\subsection{Forming quality}

The wall thickness deviation $\Delta$ and ovality $e$ are the important indexes to evaluate the dimensional accuracy of spun parts, as reported by Xia et al. [9]. Figure 5 shows the influence of total thinning ratio $\psi$ on wall thickness deviation $\Delta$, where the actual thinning ratio of the spun parts is smaller than that of the designed ones (listed in Table 1 and Table 2) due to the springback of the spun parts and the spinning machine. It shows that the wall thickness deviation $\Delta$ of the spun part decreases rapidly with 
Table 1. Process parameters of stagger spinning.

\begin{tabular}{|l|c|c|c|c|}
\hline Pass & $\begin{array}{l}\text { Thinning ratio per pass } \psi_{i} / \\
\text { Total thinning ratio } \psi_{t} / \%\end{array}$ & $\begin{array}{l}\text { Radial offset } \\
b_{12} / \mathrm{mm}\end{array}$ & $\begin{array}{l}\text { Radial offset } \\
b_{23} / \mathrm{mm}\end{array}$ & $\begin{array}{l}\text { Axial offset } a_{12} \& \\
a_{23} \\
/ \mathrm{mm}\end{array}$ \\
\hline 1 & $35 / 35$ & 0.45 & 0.35 & $2.5 / 2.5$ \\
\hline 2 & $38 / 60$ & 0.35 & 0.25 & $2.5 / 2.5$ \\
\hline 3 & $35 / 74$ & 0.15 & 0.15 & $2.0 / 2.0$ \\
\hline 4 & $43 / 85$ & 0.15 & 0.10 & $2.0 / 2.0$ \\
\hline 5 & $42 / 90$ & 0.08 & 0.06 & $2.0 / 2.0$ \\
\hline
\end{tabular}

Table 2. Process parameters of counter-roller spinning.

\begin{tabular}{|l|c|c|c|}
\hline Pass & $\begin{array}{l}\text { Thinning ratio per pass } \psi_{i} / \\
\text { Total thinning ratio } \psi_{t} / \%\end{array}$ & $\begin{array}{l}\text { Reduction of the } \\
\text { outer roller } \\
\Delta \mathrm{t}_{1} / \mathrm{mm}\end{array}$ & $\begin{array}{l}\text { Reduction of the } \\
\text { inner roller } \\
\Delta \mathrm{t}_{2} / \mathrm{mm}\end{array}$ \\
\hline 1 & $35 / 35$ & 1.05 & 1.05 \\
\hline 2 & $45 / 64$ & 0.875 & 0.875 \\
\hline 3 & $40 / 78$ & 0.43 & 0.43 \\
\hline 4 & $37 / 86$ & 0.24 & 0.24 \\
\hline 5 & $35 / 91$ & 0.14 & 0.14 \\
\hline
\end{tabular}

the increasing of the total thinning ratio $\psi$ during both the stagger spinning and counter-roller spinning. The reason is that the deformation degree in thick thickness area of the spun parts is larger than that of the thin thickness area during spinning, so that the uniformity of the wall thickness can be improved by the spinning process [9]. The deviation $\Delta$ under stagger spinning is smaller than that of the counterroller spinning. This is because that the cylindrical blank is supported by three inner rollers during counter-roller spinning, it behaves as point contact between the cylindrical blank and inner roller. While during stagger spinning, the cylindrical blank is supported by mandrel on the inner surface, therefore the stability of the stagger spinning is better than that of the counter-roller spinning, which is beneficial for improving the uniformity of the wall thickness. However, the wall thickness deviation $\Delta$ of spun parts obtained by counter-roller spinning is less than $0.1 \mathrm{~mm}$ when the total thinning ratio $\psi$ reaches at $84 \%$, and the deviation $\Delta$ of spun parts decreases rapidly with the further increasing of the total thinning ration $\psi$. As reported by Xia, to obtain the refined grain size of $600 \mathrm{~nm}$, the total thinning ratio $\psi$ should be $87 \%$ [4]. Therefore, the cylindrical parts with ultrafine grain structure and small deviation $\Delta$ can be obtained by both the stagger spinning and the counter-roller spinning.

Figure 6 shows the influence of total thinning ratio $\psi$ of wall thickness on ovality $e$ of spun parts. It shows that the ovality $e$ increases with the increasing of the total thinning ratio $\psi$ during both the stagger spinning and counter-roller spinning. The reason is that the instability of the spun parts increases with the increasing of the total thinning ratio $\psi$, which will lead to expansion of the diameter or torsion during spinning; moreover the work hardening effect will result in the decreasing of the ductility of the spun parts [10]. The ovality $e$ manufactured by the counter-roller spinning is less than that of the stagger spinning. This is because that during counter-roller spinning, the inner and outer surfaces of the cylindrical blanks deformed simultaneously under the action of the spinning forces from the inner and outer rollers individually. Therefore, the distribution of the plastic strain of the spun parts induced by counter-roller spinning is more homogeneous than that of stagger spinning, which is beneficial for the decreasing of the ovality [3]. The ovality $e$ obtained by counter-roller spinning is $0.10 \mathrm{~mm}$ when the total thinning ratio $\psi$ reaches at $84 \%$; the ovality $e$ by stagger spinning increases rapidly after the total thinning ratio $\psi$ is greater than $56 \%$, which is much greater than that of the counter-roller spinning. 


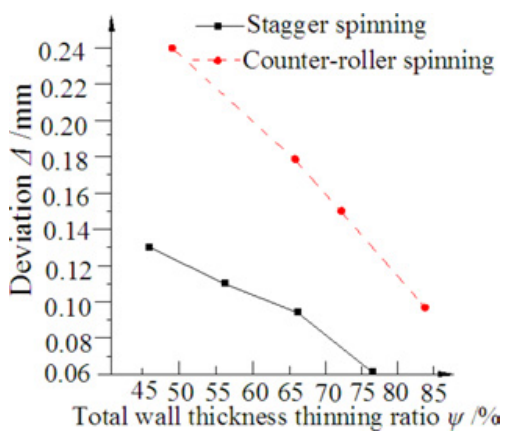

Figure 5. Influence of total thinning ratio $\psi$ on deviation $\Delta$.

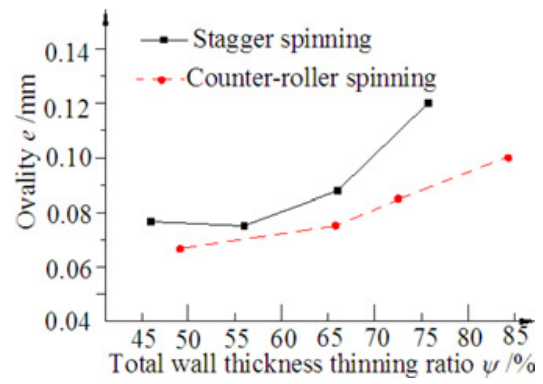

Figure 6. Influence of total thinning ratio $\psi$ on ovality $e$.

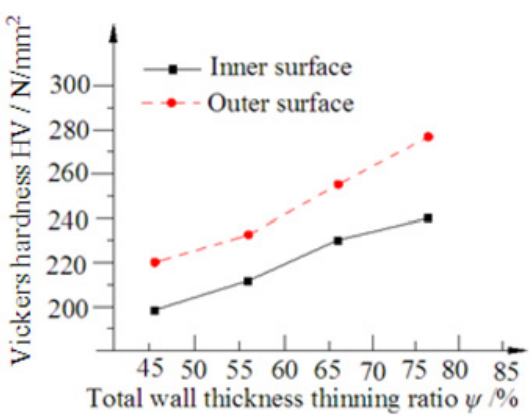

(a) Stagger spinning

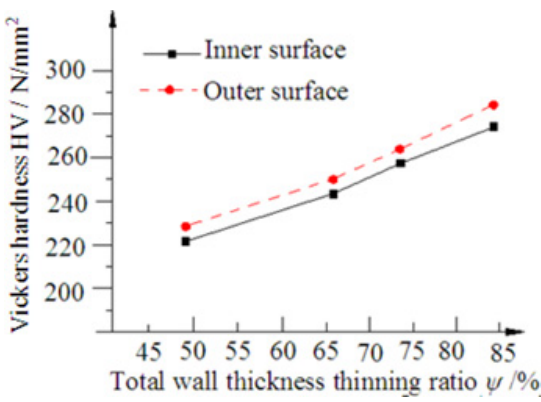

(b) Counter-roller spinning

Figure 7. Influence of total thinning ratio $\psi$ of wall thickness on hardness HV of spun parts.

\subsection{Mechanical properties}

The hardness HV, strength $\sigma$ and elongation $\delta$ are the main indexes to evaluate the mechanical properties of the spun parts [4]. Figure 7 shows the influence of total thinning ratio $\psi$ of wall thickness on hardness $\mathrm{HV}$ of the spun parts. The results show that the hardness HV increases with the increasing of the total thinning ratio $\psi$, and the hardness $\mathrm{HV}$ on inner and outer surfaces of the spun parts manufactured by counter-roller spinning is more homogeneous than that of the stagger spinning. The maximum difference of the hardness HV between the outer and inner surfaces by counter-roller spinning is $9.4 \mathrm{HV}$ when the total thinning ratio $\psi$ reaches at $84 \%$, while the maximum difference of the hardness $\mathrm{HV}$ by stagger spinning is $39 \mathrm{HV}$ when the total thinning ratio $\psi$ reaches at $76 \%$. This is because that the deformation degree on the outer surface is much greater than that of the inner surface during stagger spinning [11]. While during counter-roller spinning, the plastic strains of the inner and outer surfaces of the cylindrical blank are nearly the same [11]. The hardness HV is proportional to the deformation degree, so that the distribution of hardness of spun part obtained by the counter-roller spinning is more homogeneous than that of the stagger spinning [3].

Figure 8 shows the influence of total thinning ratio $\psi$ of wall thickness on yield strength $\sigma_{\mathrm{s}}$, tensile strength $\sigma_{\mathrm{b}}$ and elongation $\delta$ of the spun parts. It shows that the yield strength $\sigma_{\mathrm{s}}$, tensile strength $\sigma_{\mathrm{b}}$ and the elongation $\delta$ are mainly influenced by the total thinning ratio $\psi$, and have little relevance to the spinning method. With the increasing of the total thinning ratio $\psi$, both the yield strength $\sigma_{\mathrm{s}}$ and the tensile strength $\sigma_{\mathrm{b}}$ increases but the elongation $\delta$ decreases due to the effect of the work hardening. 


\section{MATEC Web of Conferences}

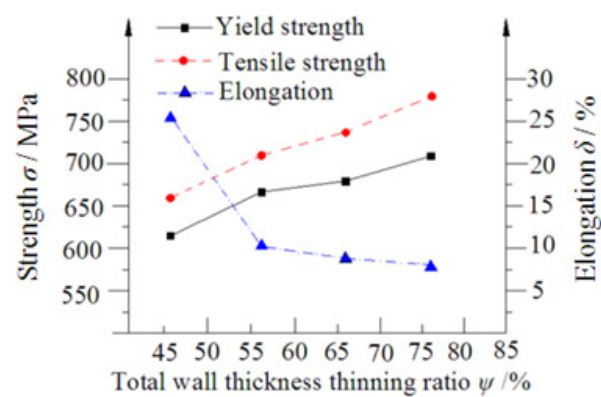

(a) Stagger spinning

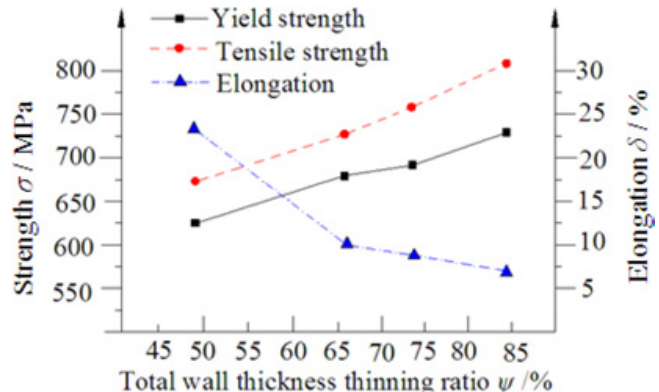

(b) Counter-roller spinning

Figure 8. Influence of total thinning ratio $\psi$ of wall thickness on strength and elongation.

The yield strength $\sigma_{\mathrm{s}}$ of the spun parts increases from $300 \mathrm{MPa}$ to $700 \mathrm{MPa}$, while the elongation $\delta$ decreases from $36 \%$ to $6 \%$ when the total thinning ratio $\psi$ reaches at $80 \%$.

\section{Conclusions}

The dimensional accuracy and mechanical properties during stagger and counter-roller spinning are studied experimentally. The following conclusions can be drawn:

1. The wall thickness deviation and ovality of the spun parts are closely related to the spinning method and the total thinning ratio of wall thickness. The wall thickness deviation of the spun parts manufactured by stagger spinning is smaller than that of the counter-roller spinning, while the ovality of the spun parts manufactured by stagger spinning is larger than that of the counterroller spinning. The wall thickness deviation of the spun parts decreases and the ovality increases with the increasing of the total thinning ratio.

2. The hardness of the spun parts increases with the increasing of the total thinning ratio of wall thickness, and the hardness on inner and outer surfaces of the spun parts manufactured by counterroller spinning is more homogeneous than that of the stagger spinning.

3. The strength and the elongation of the spun parts are mainly affected by the total thinning ratio of wall thickness, with little relevance to the spinning method, with the increasing of the total thinning ratio, the strength increases but the elongation decreases.

4. The counter-roller spinning is an effective method to manufacture the cylindrical parts with ultrafine grain structure as well as high dimensional accuracy and excellent mechanical properties.

The research team would like to acknowledge the financial support of National Natural Science Foundation of China (No: 51075153, 51375172); the Provincial Natural Science Foundation of Guangdong (No: 10151040301000000); and the EU Marie Curie Actions - MatProFuture Project (FP7-PEOPLE-2012-IRSES-318968).

\section{References}

[1] Kalpakjian S. Manufacturing engineering and technology. Pearson Education India (2001)

[2] Q.X. Xia, G.F. Xiao, H. Long, X.Q. Cheng, X.F. Sheng. Int. J. Mach. Tools Manuf. 85, 100-121 (2014)

[3] G.F. Xiao, Q.X. Xia, X.Q. Cheng, Y.J. Zhou. Int. J. Adv. Manuf. Technol. (to be published)

[4] Q.X. Xia, G.F. Xiao, H. Long, X.Q. Cheng, B.J. Yang. Mater. Des. 59, 516-523 (2014)

[5] X. Sauvage, G. Wilde, S. Divinski. Mater Sci Eng A. 540, 1-12 (2012) 


\section{ICNFT 2015}

[6] C. Zeng. Research on forming method and experiment of nanocrystalline/ultra-fine grain structure cylindrical parts by counter-roller spinning. Master's thesis of South China University of China (2014) (in Chinese)

[7] Metallic materials-Tensile testing- Part 1: Method of test at room temperature. Chinese standard Press (2010) (in Chinese)

[8] C.H. Wang, K.Z. Liu. Spinning technology. China Machine Press (1986) (in Chinese)

[9] Q.X. Xia, P. Zhang, X.Q. Cheng, B.J. Yang. Forg. Stamp. Technol. 37(6), $42-46$ (2012) (in Chinese)

[10] Q.X. Xia, S.S. Susumu, H. Kotera. J. Mater. Process. Technol. 159(3), 397-400. (2005) (in Chinese)

[11] G.F. Xiao, Q.X. Xia, X.Q. Cheng, Y.J. Zhou. Proceedings of the 11th International Conference on Frontiers of Design and Manufacturing, Nanjing, China (2014)

[12] Chen GX, Jia WD, Cao GS. Process and equipment of power spinning. Press of National Defence Industry (1986) (in Chinese)

[13] C. Zeng, Q.X. Xia, G.F. Xiao, H.X. Xie. Forg. Stamp. Technol. 39(4), 36-41 (2014) (in Chinese) 